\title{
The Development of a National Anthropometric Data Bank for Saudi Adults: The Initial Stage
}

\author{
Moudi ALMOUSA \\ College of Food Sciences and Agriculture, King Saud University, Riyadh, Saudi Arabia \\ DOI: 10.15221/16.266 http://dx.doi.org/10.15221/16.266
}

\section{Background}

Although the Saudi Arabian apparel market is the largest in the Middle East, there is no data or current size standards to accurately define the anthropometric size and shape of Saudi population. In recent years, there have been requests from some of potential investors in the apparel industry and consumers to provide relevant size reference material. There is an urgent need for such data bank especially with the country's newly announced 2030 Vision to diversify the economy by welcoming foreign investments and creating 35 industrial cities under the umbrella of Saudi Industrial Property Authority (MODON), where the localization of many industries including apparel is highly encouraged. Saudi Arabia is a developing country with an area of $2,149,690 \mathrm{KM}^{2}$. The total population is $31,015,999$, while Saudi nationals are only 20,774,906 (General Authority for Statistics, 2014). The gross domestic product per capita in 2015 is $\$ 24,400$ (World Bank, 2015).

The main aim for SizeSaudi national survey is to develop a national anthropometric data bank for Saudi adults using 3D scan technology. A sizing system for both Saudi males and females will be developed as-well-as body shape analysis for the adults Saudi nationals. The project is fully funded by King Abdulaziz City for Science and Technology (KACST). This paper presents the summery of the planning phase of the project.

\section{Survey Planning}

The preparation was undertaken during 2013 where the proposal for the project was completed and sent for (KACST) to be reviewed for possible funding. During 2014, the project got an approval for funding and the planning for the survey started. King Saud university in Riyadh, provided logistic support; providing fully equipped space to be used for offices for the research team and lab, which will be used later as a center for 3D body scanning. Ethical approval of the study protocol was granted by the Ethic Committee of King Saud University.

SizeSaudi research team setup a performance criteria for the project, which depends mainly on accuracy, reliability, available software, and after-sale services within available funding. Moreover, an extensive review of similar international projects such as SizeUSA, SizeUK, SizeChina, and SizeThailand was conducted to identify best practices in conducting national surveys with regard to methodology and equipment used. With regard to benchmarking in choosing the scanner, the team reviewed the available 3D scanners on the market according to the selected criteria. In addition to the previously mentioned criteria that comprise accuracy, reliability, available software, and after-sale services, other criteria were included in the selection process. Since the main outcome of the anthropometric 3D database is establishing a sizing system for Saudi adults, ability for the system to extract accurate measurement sets for males and females suitable for clothing industry was the major selection criteria. Three scanners were initially chosen then manufacturers were contacted to provide complete specifications for available equipment and software, provide a sample of a full body scan, and a complete quotation including hardware, software, and services.

Very few studies are available in literature regarding performance standards of the selected scanners, which make it a weak resource for the decision-making process in choosing the scanner. After reviewing available documents regarding the three scanners, the research team and the project consultant agreed on purchasing Symecad ${ }^{\mathrm{TM}}$ Survey scanner. It is based on photogrammetry technique, which works by projecting safe white light stripes on scanned subjects and capture the distortions of light stripes by cameras. The scanner has high resolution sensors with average point density of 25 points $/ \mathrm{cm}^{2}$, one second 3D scanning time, and five seconds calibration time. The automatic body measurement extraction is based on ISO-8559 standard. In addition to 140 3D body measurements that will be obtained from the scanner, manual anthropometric measurements and body composition analysis will be obtained. 


\section{Sampling and recruitment method}

Unlike similar national sizing surveys that have been conducted, SizeSaudi project may face different obstacles due to the conservative nature of the Saudi culture where privacy may be an issue especially in collecting 3D data for female participants. To solve this issue, we omitted the awareness campaigns and media advertisements that were initially proposed.

International anthropometric standards such as (ISO 7250; Basic body measurements for technological design" and ISO/DIS 15535 "General requirement for establishing anthropometric database") serve as a reliable guide for survey methodology, which include the sampling method and number of subjects needed to achieve statistical significance and the required data (Annis 1978, and Roebuck, 1995).

The country is divided into 13 administrative regions. However, for the sake of the study we divided the country into five geographical regions; Middle, East, West, North, and South. A stratified sampling method will be used according to the recommendations of ISO/DIS 15535. Five thousand Saudis adults aged 18 years or older will be scanned using three-dimensional body scanner.

A multistage data collection process will be employed according to the established protocol which is adapted from Anthropometric Standardization Reference Manual (Lohman and Martorell, 1988) and National Health Nutrition Examination Survey: Anthropometry Procedures Manual, 2007.

First, respondents will register and respond to questionnaire and book an appointment through the project website. Participants will be then contacted by an administrative person for confirmation of appointment and data collection location. Upon arrival, each participant will be greeted and invited to watch with a group an introduction video about the project and the scanning process and will be then asked to read and sign the consent form which includes a unique reference number on the first page, where participants will carry with them through all data collection stages. Participant will be moved to the next station where they will be provided a scan ware and ask to change their clothing in the changing room. The next stage is manual measurements and body composition analysis, followed by the last stage, which is 3D scanning.

Questionnaire includes sociodemographic characteristics, body satisfaction, health-related habits, and history of chronic diseases. The instrument was translated into Arabic by the research team, and then back- translated by a professional translator to ensure the accuracy of translation where some adjustments were made. A pilot was conducted on 35 respondents for test validity, and necessary adjustments were made.

After collecting data from participants, three different sets of questionnaires will be sent via email for participants who are willing to further participate in any of the sub studies including: marketing and purchase behavior, and lifestyle and dietary habits. Manual measurements that will be collected include stature, crotch height, crotch length, vertical trunk circumference, and armcye height as advised by the project expert advisor. Body Composition Analyzer will be used which provides weight and a complete body composition analysis for each participant in less than 30 seconds. Data include weight, body fat percentage, body fat mass, body mass index (BMI), fat free mass, estimated muscle mass, total body water, and basal metabolic rate. Tow static scan poses; standing and sitting, will be captured for each participant.

Data will be collected from five main regions in the country (Central, North, East, West, and South). Data for female participants will be collected in 3D scanning centers inside female university campuses and for male participants in booths in main university lobbies as well as in shopping malls.

Two training courses, one after scanner installation and the other just before starting the pilot by an expert from the 3D scanner manufacturing company for the research team on the 3D body scanner and purchased software were conducted. A series of experiments were conducted to test for synchronizing the three data sets; questionnaires, manual measurements and body composition analysis, and 3D scan data. Moreover, three samples of modest scan wear were tested to determine which scan wear will be used without jeopardizing the quality of the 3D scan data. After finishing the planning stage, a pilot study will take place in October 2016 with 50 participants, 25 females and 25 males before conducting the national survey to validate data collection procedure. 


\section{Reference}

Annis, J. F., Anthropometric Sourcebook, Volume 1: Anthropometry for Designers, 1978, Houston, USA: NASA - Reference Publication 1024.

Anthropometric Standardization Reference Manual. TG Lohman, AF Roche and R Martorell (Eds.) 1988; Human Kinetics Book, Champaign II.

(General Authority for Statistics, 2015). http://www.stats.gov.sa/en/indicators/13

International Standards Organization, Basic Human Body Measurements for Technological Design, 1996, Geneva, Switzerland: International Organization for Standardization.

International Standards Organization, ISO 15535:2006 - General Requirements for Establishing Anthropometric Databases, 2006, Geneva: International Organization for Standardization.

National Health and Nutrition Examination Survey (NHANES): Anthropometry Procedures Manual, 2007.

Roebuck, J. A., Anthropometric Methods: Designing to Fit the Human Body (CA, USA: HFES (Human Factors and Ergonomic Society), 1995).

World Bank. Available at: http://data.worldbank.org/indicator/NY.GDP.PCAP.CD?locations=SA 